\title{
Detection of the Viral Infectious Diseases in Blood by Surface-Enhanced Raman Spectroscopy: Mini Review
}

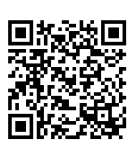

\author{
Yin-Ting Yeh* \\ Department of Physics, Material Research Institute, The Huck Institutes of the Life Sciences, The Pennsylvania State University, USA
}

Submission: November 28, 2018; Published: December 17, 2018

*Corresponding author: Yin-Ting Yeh, 104 Davey Laboratory, University Park, PA 16802, USA

\begin{abstract}
Blood is the most complex fluid in the body. Blood contains a large number and variety of cells and molecules. When viruses infect a host, biomarkers of the viral infections are rare. Thus, it is hard to detect in blood. Recently, Surface Enhanced Raman Spectroscopy (SERS) has been shown to be promising in infectious disease detection. SERS provides a sensitive, rapid, and non-invasive approach to virus detection. Several studies have demonstrated the use of SERS in detecting various types of infectious diseases. In this report, we briefly review recent advances in the detection of infectious diseases by using SERS.
\end{abstract}

Keywords: Virus detection; Raman spectroscopy; Infectious disease

Abbreviations: LSPR: Localized Plasmon Resonance; SERS: Surface-Enhanced Raman Spectroscopy, PCA: Principal Component Analysis, HCA: Hierarchical Cluster Analysis

\section{Introduction}

Raman spectroscopy is an optical spectroscopic technique that is commonly used to identify the vibrational modes of a substance. However, the efficiency of exciting Raman scattering is very weak-approximately 1 out of $10^{6}$ phonons are absorbed and emitted through Raman scattering. This weak efficiency dramatically limits the signal intensity of Raman spectroscopy. Studies have shown that metal nanoparticles can dramatically enhance Raman scattering via a nanoparticles-based localized plasmon resonance (LSPR). This enhancement of the Raman signal enables detection down to the single molecule level ( picomolar) [1,2]. Surface-enhanced Raman spectroscopy (SERS) is a technique which induces local surface plasmons at the vicinity of a metal or dielectric material by laser excitation to enhance the Raman signal. This surface-based detection technique can enhance the Raman signal up to $10^{10}$ by molecular adsorption on a rough metal surface and makes single biomolecular detection possible if the target molecule is near the metal surface $[3,4]$. When detecting viruses there are two conventional approaches to bringing the target virus close to the rough metal, one is through antibody-conjugated nanoparticles, and the other is through surface engineered metal nanostructures. In the following, we will focus on reviewing SERS based detection for different infectious diseases.

\section{Discussion}

Silver nanorod array substrates for SERS were prepared by Shanmukh et al. for label-free detection of different types of viruses [5]. This functionalized substrate could provide signal enhancement up to $10^{8}$ when the size of the nanostructure matches with the virus. Their spectrum analysis results showed that different RNA viruses could be differentiated by the SERS spectra. Furthermore, different strains of the same type of virus can be distinguished by the intensities of the peaks in the 900$700 \mathrm{~cm}-1$ range of the SERS spectrum. They further introduced a multivariate statistic technique for robust virus strain and type diagnosis. By principal component analysis (PCA) a closely related strain of influenza can be differentiated with $10^{3} \mathrm{PFU} /$ $\mathrm{mL}$ and further, by hierarchical cluster analysis (HCA), a single gene deletion can be detected [6]. In another study, an aptamerfunctionalized SERS substrate was used for influenza viral nucleoprotein detection [7]. Their results showed that SERS could be used for detection, identification, and classification of the binding of influenza nucleoproteins to aptamers. A single strand DNA aptamer modified silver nanorod substrate was used for the SERS-based profiling of RNA expression of the genetic mutation in the influenza PB1-F2 protein which is related to influenza virulence and pandemic potential. Using multiple HCA and partial least squares regression, their results showed $100 \%$ accuracy of complementary virus RNA target detection compared to the non-complementary RNA sequences, and the detection limit reached $10 \mathrm{nM}$ [8].

In order to acquire repeatable and high signal-to-noise ratio Raman spectra, surfaces with high order and uniform nanostructures with low fabrication variation are required. 
Chang et al. used a pyramid diamond tip of $20 \mathrm{~nm}$ radius to fabricate a gold nano-cavity array on a silicon surface with 200 nm gold deposition [9]. Lin et al. patterned a gold-coated surface with different geometries with dimensions ranging from 150 to $300 \mathrm{~nm}$ by using a focused ion beam [10]. They demonstrated that repeatable Raman spaectra could be obtained for different types of virus and concluded that when the dimension of the nanostructure matches with the target virus dimension, better signal-to-noise ratios can be obtained [11]. In another study, a commercially available SERS-active substrate was used for the rapid discrimination of poxviridae virions [12]. By using a multivariate calibration method, the Raman spectrum could be used to discriminate the unknown parapox virus.

Using surface functionalized nano metal particles, Raman signal of the targeted virus could be selectively enhanced within a suspension containing multiple viruses. Combining antibody conjugated magnetic and $\mathrm{Au}$ nanoparticles, virus bound complexes can be concentrated through an external magnetic field and detected by SERS. Another method involved a sandwich immunoassay of viruses based on surface-enhanced Raman scattering and was established by Neng et al. [13]. Paramagnetic nanoparticles (PMPs) were used for separation and enrichment of the virus, and the Raman reporter-coated Au nanoparticles (GNPs) were used as Raman signal reporters. By using this platform, the antigens of West Nile virus and Rift Valley fever virus in PBS could be detected with an approximately $5 \mathrm{fg} / \mathrm{mL}$ detection limit.

\section{Conclusion}

SERS based detection platforms provide a non-invasive, labelfree approach and also fulfill the point-of-care requirements of rapidness and potential user-friendliness. However, when dealing with a wild-type sample, there are variances of the background noise. It is important to enhance the signal-tonoise ratio to reduce false positive or negative diagnosis. Compared with other detection platforms, optical detection can be challenging to miniaturize mainly due to the bulkiness of various optical components. However, developments in fiber optics, optoelectronics, and photonics have significantly shrunk component sizes. It is likely that future optical detection systems can be more compact. Meanwhile, miniaturized, portable and sensitive Raman spectrometers will continue to develop for point-of-care applications in the future.

\section{References}

1. Ding SY, Yi J, Li JF, Ren B, Wu DY, Panneerselvam R, Tian ZQ (2016) Nanostructure-based plasmon-enhanced Raman spectroscopy for surface analysis of materials. Nature Reviews Materials 1(6).

2. Schlucker S (2014) Surface-Enhanced Raman Spectroscopy: Concepts and Chemical Applications. Angew Chem Int Ed Engl 53(19): 47564795 .

3. Kneipp K,Wang Y,Kneipp H,Perelman LT,Itzkan I,Dasari RR,Feld MS (1997) Single molecule detection using surface-enhanced Raman scattering (SERS). Physical review letters 78(9): 1667.

4. Le Ru EC, Blackie E, Meyer M, Etchegoin PG (2007) Surface Enhanced Raman Scattering Enhancement Factors: A Comprehensive Study. The Journal of Physical Chemistry C 111(37): 13794-13803.

5. Shanmukh S, Jones L, Driskell J, Zhao Y, Dluhy R, Tripp RA (2006) Rapid and sensitive detection of respiratory virus molecular signatures using a silver nanorod array SERS substrate. Nano Letters 6(11): 2630-2636.

6. Shanmukh S, Jones L, Zhao YP, Driskell JD, Tripp RA, et al. (2008) Identification and classification of respiratory syncytial virus (RSV) strains by surface-enhanced Raman spectroscopy and multivariate statistical techniques. Anal Bioanal Chem 390 (6): 1551-1555.

7. Negri P, Kage A, Nitsche A, Naumann D, Dluhy RA (2011) Detection of viral nucleoprotein binding to anti-influenza aptamers via SERS. Chem Commun 47(30): 8635-8637.

8. Negri P, Dluhy RA (2013) Detection of genetic markers related to high pathogenicity in influenza by SERS. Analyst 138(17): 4877-4884.

9. Chang CW, Liao JD, Shiau AL, Yao CK (2011) Non-labeled virus detection using inverted triangular Au nano-cavities arrayed as SERS-active substrate. Sens. Actuator B-Chem. 156(1): 471-478.

10. Lin Y-Y, Liao J-D, Ju Y-H, Chang C-W, Shiau A-L (2011) Focused ion beam-fabricated Au micro/nanostructures used as a surface enhanced Raman scattering-active substrate for trace detection of molecules and influenza virus. Nanotechnology 22(18): 185308.

11. Lin Y-Y,Liao J-D,Yang M-L,Wu C-L (2012) Target-size embracing dimension for sensitive detection of viruses with various sizes and influenza virus strains. Biosens Bioelectronics 35(1): 447-451.

12. Alexander TA (2008) Development of methodology based on commercialized SERS-active substrates for rapid discrimination of Poxviridae virions. Anal Chem 80(8): 2817-2825.

13. Neng J, Harpster MH, Wilson WC, Johnson PA (2013) Surface-enhanced Raman scattering (SERS) detection of multiple viral antigens using magnetic capture of SERS-active nanoparticles. Biosens Bioelectron 41: 316-321. 


\section{Your next submission with Juniper Publishers} will reach you the below assets

- Quality Editorial service

- Swift Peer Review

- Reprints availability

- E-prints Service

- Manuscript Podcast for convenient understanding

- Global attainment for your research

- Manuscript accessibility in different formats ( Pdf, E-pub, Full Text, Audio)

- Unceasing customer service

Track the below URL for one-step submission https://juniperpublishers.com/online-submission.php 